\title{
THE POLYTHENE PACK OPERATION FOR PULMONARY TUBERCULOSIS
}

\author{
BY

\begin{abstract}
H. MORRISTON DAVIES, LESLIE J. TEMPLE, AND CHRISTOS STATHATOS
From the Liverpool Thoracic Surgical Centre, the Department of Surgery,
\end{abstract} \\ Liverpool University, and the Cheshire Joint Sanatorium
}

(RECEIVED FOR PUBLICATION MARCH 28, 1951)

Surgical collapse of the lung has won an honoured place in the treatment of pulmonary tuberculosis and, in the form of thoracoplasty, is widely used. We are, however, very conscious of the drawbacks of this operation. No matter what the individual technique or aftercare, in a certain proportion of cases the collapse of the apex of the lung, which had seemed so perfect a week after operation, is found a few weeks later to be less complete, and cavities which had closed to have reopened in the re-expanding lung. The ordeal of multiple stages and the inevitable permanent deformity are, moreover, distasteful to the surgeon and to the patient alike, being acceptable only as a life-saving necessity.

In the past, efforts have been made to overcome these defects by the use of plombage. The experience at that time of one of us (Morriston Davies, 1933, 1944) was not encouraging. Paraffin plombs had a tendency to lead to ulceration from surface cavities, or to produce tissue reaction leading to abscess formation necessitating the subsequent extraction of the foreign body. Living tissue grafts of breast and muscle failed because they were difficult to pack into the extrapleural space and, even when the immediate result was good, later shrank. Plombage, with fat from other parts of the body and lipomata, was equally unsatisfactory owing to the tendency to liquefy and disintegrate. Later extrapleural pneumothorax was tried and still has its advocates, as has the substitution of the air in the extrapleural space by oil.

Since 1937 Semb has been advocating the extrafascial stripping of the lung from the mediastinum when doing a thoracoplasty. For a time this was hailed by some as a solution of the problem of re-expansion of the lung but not of the deformity. Still later Semb (1950) tried air refills of the "Semb space," and he and others (Edwards, 1949) have attempted many varieties of bone graft and suture to assist in maintaining the collapse of the lung and cavity. The quest for a solution of the two problems, the prevention of re-expansion and of deformity associated with a thoracoplasty, continues.

In 1946 Wilson published his paper on the use of polymethyl methacrylate or lucite balls as an extrapleural plomb. This interested us as there was evidence that the balls did not irritate the tissues, and it seemed evident that they could be made to accommodate any size and shape of space. When, however, we obtained samples of these lucite balls we decided against their use. The very hardness and unresilient qualities of lucite, at any rate as spheres, seemed to us to constitute a danger. The insertion of an unyielding substance extrapleurally in apposition to the lung, with 
possibly a cavity or ulcerative changes close to the surface, is to invite the risk of ulceration extending through to the pleural cavity, and of infection. The late results of the Wilson operation in America have shown these fears to be justified (Trent, Moody, and Hiatt, 1949).

We felt, none the less, that the possibilities of using some form of plastic were worth exploring. After experiments with various types of these materials one of us (H. M. D.), in conjunction with Dr. Wevill and Mr. Holt, of Imperial Chemical Industries, became interested in polythene sheeting, from which the idea of the polythene bags filled with strips of polythene sheeting was evolved. The product of this combination was a pack which was firm, resilient, but, while not too easily compressible, could be moulded to the shape of the space it had to fill and was efficacious in controlling the collapse of the lung. While investigating the possibilities of plastic materials we also became interested in their application, not as an extrapleural plomb, but of their insertion into an extra-periosteal-muscular space. There the foreign substance would be separated from the lung by pleura, intercostal muscles, and periosteum, and be bounded outside by the ribs partially denuded of periosteum. In common with all extrapleural operations this procedure has the advantage over the thoracoplasty that there is no resultant deformity of the chest.

It was obvious that a considerable amount of experimental work would have to be done as a preliminary to the actual operation. The effect of the plastic on the tissues had to be determined, as also the results of leaving in situ ribs which had been partly or wholly denuded of periosteum. There were in addition many difficulties to be overcome and mistakes had to be rectified before an established procedure was adopted. It was in fact nearly two years before the present technique for the polythene pack operation became established.

\section{EXPERIMENTAL WORK}

In our experiments the first substance tested was polymethyl methacrylate powder. Powder was used as it could fill the space without undue pressure at any point. The right pleural cavity of a rabbit was opened and some of this powder inserted. The rabbit was killed after an interval of four months, and no trace of powder was found. There were adhesions of the lung to the chest wall. The whole lung and chest wall were sent for histological examination. The section showed the pleural surface of the ribs to have lung adherent to it and, between the two, giant cells surrounded by fibrosis. There were also giant cells deep to the visceral pleura, and in some of these crystals or fragments of a doubly refractile matter could be seen.

Our attention was next turned to polyethelene, more commonly known as polythene. Considerable experimental work has been published showing that polythene is non-irritant in the tissues (Ingraham, Alexander, and Matson, 1947 ; Farquer and Lewis, 1948 ; Grindlay, 1948), and polythene has been used as a plastic in skull defects (Brown, Grindlay, and Craig, 1948). Earlier work produced a certain confusion and contradiction, subsequently shown to be brought about by the presence of a stripping agent, dicetyl phosphate, upon the surface of the film (Yeager and Cowley, 1948). In the present work polythene film manufactured from pure polythene by a "melt" casting, as distinct from a "solvent" casting process, was employed, and the surface of the film was carefully washed before use. Chemically it consists of 500 or more linked ethylene units and is insoluble in all solvents at 
temperatures below $60^{\circ} \mathrm{C}$. It is resistant to strong acids or alkalis and has no interaction with saline, glucose, blood, sulphanilamide drugs, penicillin, or streptomycin. Its specific gravity is 0.92 and its melting point $115^{\circ} \mathrm{C}$. It can be boiled but not autoclaved. It has high dimensional stability, is highly flexible, a poor heat conductor, and completely non-opaque to radiographs.

The possibility of making balls similar to the lucite balls used by Wilson was explored, but both the solid and the hollow balls proved to be just as hard and unyielding as was lucite, and all the pliancy of sheet polythene was lost when in a spherical form.

We tried the effect of placing solid polythene in human subcutaneous tissue for two months and found no foreign body reaciion or change in the polythene.

The effect of leaving ribs in situ but denuded of periosteum was also investigated. The immediate results of Bailey's (1942) and Paneth's (1946) operations in which they left ribs in situ denuded of periosteum were encouraging. On two occasions at a first stage thoracoplasty the fourth rib was denuded of periosteum and was left in situ. This rib was removed, in one case five and in the other two weeks later, when doing the second stage. There was no gross evidence of necrosis or infection, nor of any adverse clinical reaction.

Experiments were now undertaken using polythene sheeting 3/1,000 of an inch thick. Rabbits were used for these experiments: it proved quite impossible to imitate the proposed operation, because the periosteum and pleura were so fragile that they were invariably torn. We made a practice, under endotracheal anaesthesia, of opening the thorax and completely stripping the fourth, fifth, and sixth ribs of their periosteum from the transverse process to the costal cartilage. A piece of polythene film folded into a small pack was then inserted between the lung and denuded ribs.

A rabbit operated on in this way was still alive two years later. Radiographs taken at intervals showed no evidence of change in the denuded ribs. Rabbits similarly treated were killed at intervals from five weeks to a year. The findings were fairly uniform. The piece of polythene was always found encased in a thin layer of fibrin which sometimes extended between the layers of polythene: in every case the polythene could be easily removed unaltered and without adhesions to it. The lung was collapsed but appeared normal otherwise, and such adhesions as existed were usually on the periphery of the polythene pack and were consistent merely with the performance of a thoracotomy. Sometimes there was some bony regeneration internally to the pack from the displaced fragments of periosteum stripped off the rib. It was difficult, except by counting, to know which were the ribs which had been stripped. In every case muscle had adhered to them, and they appeared to be of normal gross appearance, except that the overlying tissues could be stripped off fairly easily. A typical report on the histology of one of these lungs is that of a rabbit killed at 17 weeks.

\footnotetext{
"The lung is partially collapsed and has some thickening of the pleural surface. Sections show that the lung is only partially aerated, but there is no inflammatory change or foreign body reaction present. The thickened pleura consists of a loose network of fibrin which is acellular."
}

In one case only was there a suggestion of foreign body reaction and this in a rabbit which was killed at five and a half weeks. The ribs were somewhat roughened, and it would seem that in the early stages more reaction could be looked for than 
later on. The removed ribs were then examined in various ways. Some were taken for calcium estimation, using normal ribs from the same rabbit as a control. It was found that even with normal ribs the results varied by as much as $50 \%$ dependent upon which part of the rib was taken for ash: the differences between normal rib and stripped rib were always less than the range of error thus estimated. It was felt that no great significance could be attached to this part of the investigation. Section of the ribs, too, proved to be difficult of interpretation, and this applied equally to the human stripped ribs examined. In no case was there any evidence

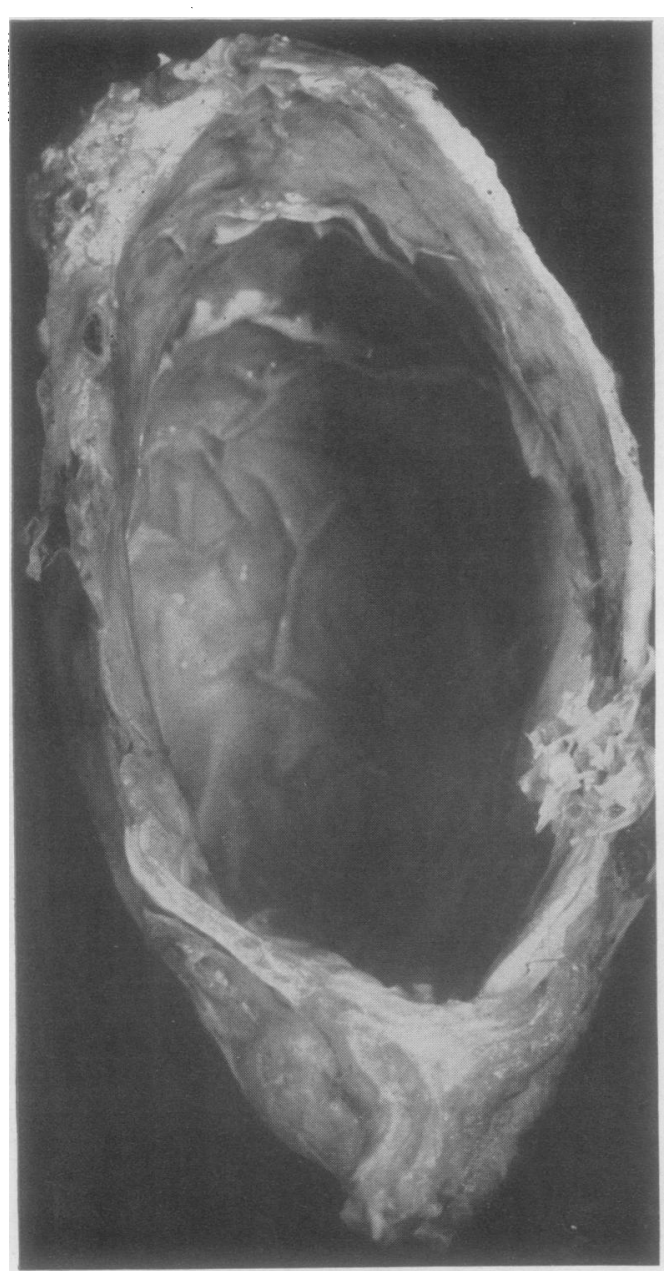

FIG. 1.-Post-mortem specimen of chest wall and mediastinal covering in patient who died of Hodgkin's disease 13 months after operation. The pack has been removed and the smooth, glistening lining reduplicating the folds in the pack is clearly seen. of necrosis, and in many the ribs appeared to be normal. This was particularly so in the ones that had been left longest before killing the animal. In some, however, changes were found suggesting that replacement of the rib was occurring. There were several areas of marked osteoclasis together with deposition of new bone. We gained the impression that the ribs were treated as bone grafts revascularized from the overlying muscles and that the bone was being replaced by new bone of exactly the same form. This would be in accord with the current findings on the use of living bone grafts.

The following conclusions may be drawn from this limited investigation. (1) That in most cases there is no foreign body reaction excited by the polythene and that ulceration into the lung does not take place even when the lung is compressed by polythene in direct apposition to it. (2) That rib stripped completely of its periosteum does not necrose, and is adequately revascularized by the overlying muscles and from vessels in the ligaments of attachment to the vertebral bodies. (3) That calcification and ossification in the stripped periosteum displaced internally to the pack can take place.

In drawing these conclusions it should be noted that the rabbit lungs investigated were normal and not tuberculous lungs. 
Inspection of the pack site in uninfected cases was possible in only two patients who died later of concomitant disease. In one patient, following a pneumonectomy for an undiagnosed neoplasm of the lung, the resulting space was obliterated by the insertion of a polythene pack in the usual manner between the periosteal beds and the bared ribs. The patient later developed typical Hodgkin's disease and died 13 months after the operation. At necropsy the pack was removed together with the overlying chest wall and the bed of the pack. There was no fluid, and the smooth lining of the space was moulded round it, repeating all its folds. Radiographs of the specimen showed only a small amount of rib formation deep to the pack. There was no evidence of any foreign body reaction, granulation tissue, or other sign of irritation (Fig. 1). In the second patient, a year after a successful polythene pack operation for collapse of the tuberculous lung, a cerebral neoplasm developed from which he died. The diagnosis was confirmed at necropsy, and the pathologist reported that the pack lying in the extraperiosteal space was dry and with no signs of irritation around it. The lung was well collapsed, there were no patent cavities, and the tuberculous areas appeared to be well fibrosed and healing. It is noteworthy that the regeneration of bone from the displaced periosteum has been disappointing. This may be due to a reduction in blood supply by division of the intercostal bundles. A further factor is the immobilization of the pack bed resulting in a lack of stimulus to bone formation.

The Making and Sterilization of Polythene Packs

Two sets of polythene bags, differing in shape and size, are supplied by Imperial Chemical Industries. The bags are made of polythene sheeting 3/1,000 of an inch thick. Each set consists of an inner and an outer envelops. The set for the apical pack is conical in shape. The inner envelope is $7 \frac{3}{4}$ in. long and $6 \frac{1}{4}$ in. broad at the base. The outer envelope is $9 \frac{3}{4}$ in. long and $7 \frac{1}{2}$ in. broad at the base. The set for the basal bags is rectangular: the inner envelope is 7 in. by $4 \frac{1}{4}$ in., the outer 9 in. by $5 \frac{1}{4}$ in.

The inner envelope of the apical set is sealed at the base and along each side, but leaving an opening at the apex $2 \frac{1}{4}$ in. in diameter. The outer envelope is closed at the apex, but the whole length of the base is left open. The inner envelope is filled to capacity with yards of $1 \mathrm{~cm}$. broad polythene ribbon and is then inserted into the outer envelope. Lipiodol is trickled in with the polythene ribbon, some $5 \mathrm{ml}$. into the larger, less into the smaller packs. The reason for this is not so much to make the packs opaque to radiographs as to maintain the softness of the polythene pack during and after boiling. Without the oil it is found that the polythene loses some of its pliability during boiling (Fig. A).

The same method is used in making the basal packs. Some are filled to capacity, others are only half filled: these are used to pack into odd spaces. The packs are sterilized by boiling for 20 minutes. To ensure submergence they are placed

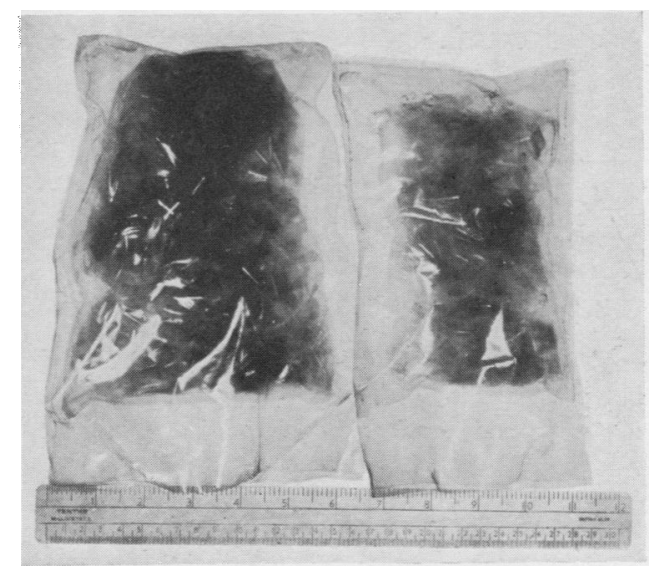

FIG. A.-Photograph of an apical and basal polythene pack. 
between two kidney dishes which are tied together. Immediately before use as much water is drained off as possible and then the packs are squeezed in a sterile towel.

\section{The Polythene Pack Operation}

This operation entails the stripping of the parietal pleura together with the intercostal muscles and periosteum from the inner surface of the first five ribs, or of six, seven, eight, and rarely nine ribs, according to the extent of the disease. The operation is always done in one stage. No ribs are removed but the third (or fourth) rib may be divided posteriorly to facilitate access to the apex and the introduction of the packs. At the end of the operation the divided surfaces of the rib are brought into apposition and sutured. In all cases the periosteum is stripped from the ribs as far back as the head and, for the first three, forward to include the perichondrium.

A light general anaesthesia has been used in combination with relaxant drugs. In the induction, following a test dose of tubocurarine or flaxedil, a dose sufficient to prevent straining and coughing at intubation is given. This varies between 20 and $30 \mathrm{mg}$. tubocurarine. This is followed by thiopentone. A cuffed endotracheal tube is introduced and anaesthesia is then maintained with $50 \%$ nitrous-oxide-oxygen and fractional doses of thiopentone. Further doses of tubocurarine are given so that respiration is controlled throughout the operation. This is also most essential at the time when the gauze packs are removed preparatory to inserting the polythene pack: at this stage complete quiescence of the mobilized lung is essential. Secretions, if present, are controlled by use of the "head-down" position and by suction. At the end of the operation prostigmin is given to ensure immediate reversal of curarization. Patients are conscious and able to cough on leaving the theatre.

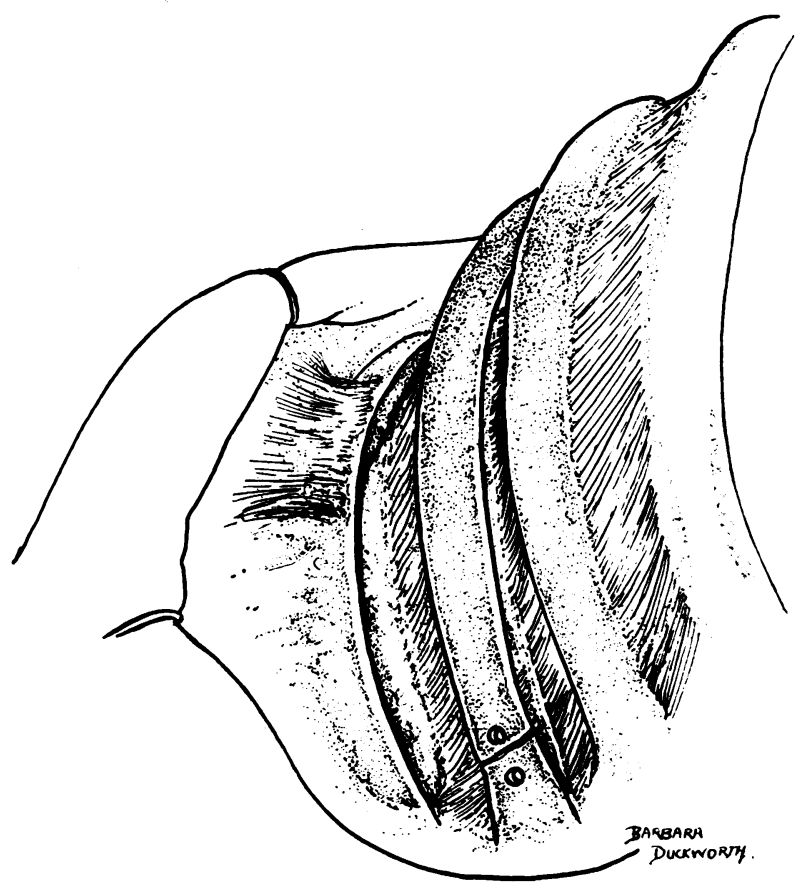

FIG. B.-First stage of the operation with upper and lower edges of the third rib freed of periosteum and intercostal muscle. The back end of the rib is divided and doubly punctured. The lower edge and under-surface of the second rib have been freed of periosteum. The intercostal bundle is still intact. The attachment of the serratus magnus muscle to the second rib is not interfered with. 
The usual " $L$ " shaped incision as for a thoracoplasty is made and is curved well forward below the angle of the scapula. The muscles are divided and the ribs are exposed. The second rib is recognized by the large attachments to its surface of the serratus magnus muscle, which must at no stage be interfered with.

With the electro-cautery the periosteum and external intercostal muscles are divided at either edge of the third rib as far forwards and backwards as possible. With a periosteal elevator the internal intercostal is detached from the upper and lower borders of the rib for about $5 \mathrm{~cm}$. and, keeping the elevator close to the bone, the periosteum is separated off the deep surface. Using the elevator as a protection to the underlying pleura and lung, the internal intercostal can be detached from the bone by scissors, when the periosteum and intercostals will fall away from the ribs (Fig. B). The separation is continued forward so as to strip the under-surface of the costal cartilage as well as the rib and then carried back until it reaches the body of the vertebra. Strips of gauze are packed under the whole length of the rib to prevent any lung movement and to help haemostasis. The same procedure is carried out with the fourth rib, except that it is not necessary to strip the cartilage.

The second rib is next stripped and once again care must be taken not to interfere with the attachments of the serratus magnus muscle to the outer surface.

At some point after this it may be advisable to divide the third rib so as to obtain better access. (It is not always necessary as, for instance, when there is wide separation of the ribs.) The third rib is divided as far back as it conveniently can be and by an oblique line of section running from below upwards and backwards. This is because there is a tendency for the anterior part of the rib to drop downwards. Before dividing the rib a puncture hole is made about $1 \mathrm{~cm}$. on either side of the proposed line of division and a separate double nylon threaded through each. It will now be possible to swing the anterior end of the third rib upwards or downwards and so get easy access to the apex (Fig. C).

Fig. C.-Periosteum has been stripped from the under-surfaces of the first and second ribs, and the upper parts of the mediastinal structures are exposed. The first and second intercostal bundles are divided posteriorly. The third rib is retracted and the edges of the fourth rib have been cleared as well as the upper border of the fifth. The lung has dropped down.

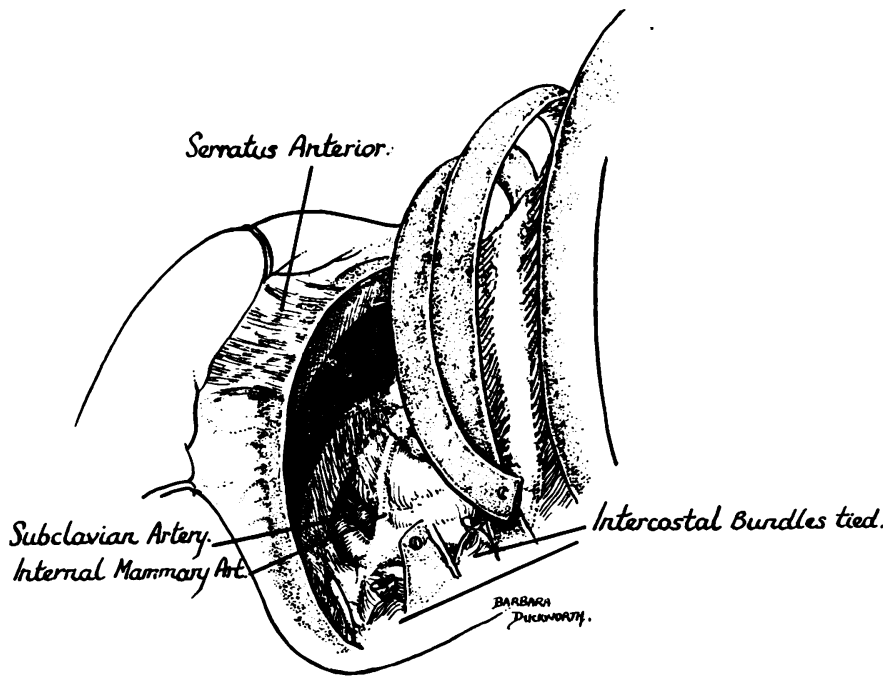

A broad copper spatula is next inserted between the fourth and fifth ribs, under the fourth rib, which helps to steady it and act as a fulcrum, up to the second rib and interspace. This helps to keep the apex of the lung steadily controlled and out of the way.

The separation of the intercostal muscles of the second interspace and of the periosteum from the under-surface of the first rib in a single sheet is not easy. It must be done as far as possible with the periosteal elevator. The exposure of the first and 
second cartilages is essential, as is the detachment anteriorly of the intercostal muscles so that they can drop away from the ribs and cartilages. At one point it will be found that muscle bundles from the pectoralis minor muscle have to be divided with scissors. Of equal, or even greater, importance is the transverse division of the freed intercostal muscles and of the neurovascular bundles as far posteriorly as possible. Unless this is done in these interspaces and later in the succeeding interspaces, it is impossible to get the lung displaced out of the costo-vertebral gutter and replaced by the polythene pack, which is essential for success.

A further essential is the exposure of the upper and inner edge of the first rib and then an extrafascial strip so as to expose at least $1 \mathrm{~cm}$. of the subclavian vessels and the brachial plexus. One of us (L. J. T.) as a routine carried the extrafascial strip down to the aorta or the azygos vein.

The apex of the lung is now free and separated from all bony structures. The space is packed with gauze to control movements of the lung and to help haemostasis, and the spatula is withdrawn.

The operation is continued to include the fifth rib, dealt with as is the fourth. If necessary the sixth, seventh, and eighth are dealt with in the same way. One point to be noted is that, if the equivalent of a six-rib or seven-rib collapse is needed, it is only necessary to strip the upper edge of the sixth rib or of the seventh. In every case, however, the intercostal bundles at the posterior end must be divided. When this is completed gauze is packed under the ribs and into the costo-vertebral gutter while the packs are made ready.

For a five-rib thoracoplasty a conical pack and a full rectangular pack will be required and, perhaps, a "half" pack. The gauze is taken out, but at the same time the copper spatula is reintroduced to maintain control of the lung. A final look round is made to ensure that there are no muscle or other attachments which will prevent the complete collapse of the lung and overlying intercostal muscles and intervening periosteal

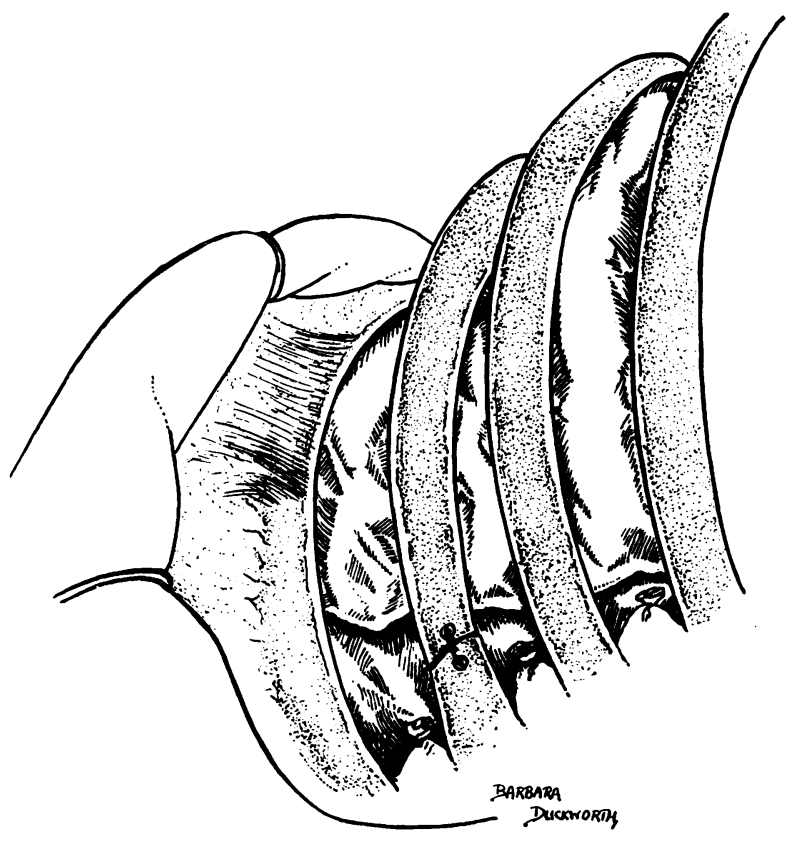

Fig. D.-The operation completed: the divided posterior ends of the intercostal bundles can be seen. The polythene packs are in situ: a half-pack fills the costovertebral gutter. The third rib has been sutured. 
strips. The whole space is dusted with procaine penicillin. The basal pack is put in first through the space made available by swinging the third rib upwards or downwards, and firmly pushed downwards and forwards. As it is inserted the spatula is removed. The apical pack is then put in, care being taken that it fits well and firmly up to the apex and forwards (Fig. D). These two packs may fill the whole space, but if not a "half" pack or even a whole rectangular one may be needed to pack into the costovertebral gutter. The packs must fit up into the apex, well forward under the costal cartilages of the first three ribs, and well back into the gutter. But in the process of fitting in the packs it is important to ensure that there is no undue pressure on, or displacement of, the mediastinum.

The divided third rib is now sutured into apposition and the wound closed in layers with interrupted thread sutures.

When a six-, seven-, or eight-rib thoracoplasty is required, the procedure is the same as described above, the lower ribs being treated like the fifth. More rectangular packs will be needed so as completely to fill the space produced by the greater displacement of the lung, and these all go in before the apical pack, and all are introduced through the gap made by the divided and displaced third rib. When inserting these basal packs one special precaution is necessary. These packs, if pressed in too tightly, may embarrass the heart. The pulse and the blood pressure must be closely observed by the anaesthetist. Any sudden alteration is an indication for immediately easing the too great pressure of the packs.

A contralateral artificial pneumothorax is no contraindication to a five-rib pack operation provided the A.P. is not diminishing the vital capacity to a serious extent. A homolateral A.P. must have been abandoned and the air completely absorbed before introducing a pack. It is also advisable to abandon a pneumoperitoneum lest, with the subsequent descent of the diaphragm, re-expansion of the lung again opens up a cavity below the pack.

Various modifications of this operation are in use by others. We understand that Professor Kremer, of Frankfurt, has for many years used an extra-periosteal operation similar to that described by Paneth and fills the space with a paraffin plomb. More recently Lucas and Cleland (1950) have adapted the operation described here, using lucite balls instead of polythene packs.

\section{RESULTS}

From April, 1948, until September, 1950, 102 polythene pack operations on 97 patients with tuberculous parenchymal disease were carried out by us. The ages of the patients were from 17 to 51. In five patients a pack was inserted on both sides. Nine had a contralateral artificial pneumothorax at the time of the operation.

The patients treated by the polythene pack operation were not specially selected. In the earlier months every case presented for a thoracoplasty which would have been submitted to this operation was treated by the insertion of a polythene pack. Several patients who had been rejected for thoracoplasty were also treated ; they agreed to take an unknown risk. The operation was also used in an endeavour to close large tension cavities, lower lobe cavities, and residual cavities after several attempts at closure by thoracoplasty had failed. It is only in the last year, with increasing experience, that the cases have been more precisely selected. As a result of this policy it has been rather the less favourable cases and those with bilateral disease that were submitted to a polythene pack operation.

In this series of 97 patients there have been two deaths attributable to the disease and these will be discussed later. 
There have been five instances of cavities in the apex of the lower lobe in all of which there was failure to render the sputum free of tubercle bacilli. These, we have learned, are unsuitable for a pack operation and therefore are excluded from the 97 patients.

In our opinion, clinical and radiological evidence of cavity closure is insufficient proof of success and we consider that sputum conversion to negative is the essential criterion of a successful operation. This has proved the only objective test.

Seventy-two of the 92 cases with predominantly upper lobe disease were successful. This gives a rate of sputum conversion of $80 \%$ of the survivors, or $78.2 \%$ if we include the two deaths. In each of these 72 patients there is now a negative sputum or no sputum at all. In 66 of these the sputum has been proved negative on repeated cultures or guinea-pig inoculation tests carried out on the sputum or, when this was not available, on examination of laryngeal swabs or gastric contents. In the other six there is no sputum, and it has not proved practicable to obtain laryngeal swabs or do gastric lavage, but on clinical grounds we are regarding these six also as successes. In these patients the period of observation varies from three months to two and a half years, during which time the sputum has been repeatedly tested.

There were 18 patients who retained a positive sputum and, of these, 11 had a cavity in the opposite lung. If we exclude these 11 cases in which we consider that the failure to obtain sputum conversion is due to a contralateral cavity, then our success rate is $88.8 \%$.

In the remaining seven there is no evidence of apparent contralateral active disease, and we must therefore assume that the disease has not been successfully controlled by the pack, although in only two of these can the cavity be demonstrated radiologically.

There were five patients on whom a bilateral operation was carried out, and three of these have been classified as successes. Of the other two, one had a cavity in the apex of the lower lobe on the second side to be treated; the cavity was not controlled by the pack. Later the pack was removed and resection of the affected segment in one stage was successfully carried out. In the second one it was found that, owing to a previously abandoned extrapleural pneumothorax, an adequate operation on the second side was not possible.

In this series there are also included three instances where operation was undertaken for a cavity persisting after a thoracoplasty ; of these two are classified amongst the successes. In the third one conversion was achieved and persisted until recently, when a cavity appeared in the opposite apex and the sputum became positive. It is intended that a polythene pack shall be inserted in the contralateral side.

There are two late deaths out of the successfully converted cases, one due to a carcinoma of the cerebellum 12 months afterwards (the post-mortem findings have been mentioned in the experimental part of this paper), and another due to a carcinoma of the larynx 20 months later, both confirmed pathologically. There have been no other late deaths in the whole series.

\section{COMPLICATIONS}

Two patients developed atelectasis of the homolateral lower lobe following operation. One of these subsequently developed a spread of disease into the lower 
lobe. Rè-expansion took place in both and, in the case of the spread, there was complete resolution following streptomycin treatment and phrenic crush. This patient has remained healed after two years. Spread of the disease did not occur in any other case.

In the early post-operative films of almost all cases a layer of fluid is to be seen outlining the lower border of the pack. The average depth of this layer is $1 \mathrm{~cm}$. In the subsequent weeks the fluid disappears. In some cases, however, there has been excessive fluid formation, and this has proved a most troublesome complication necessitating at times repeated aspiration or even removal of the pack. In eight cases the fluid formation was sufficient to require repeated aspirations, but as infection was avoided removal of the pack was unnecessary. In two of these, the wound gaped, necessitating resuture, and complete healing resulted. The final outcome was satisfactory in all these cases.

The total number of packs removed for all causes is 17 . In five, during the early post-operative period, despite aspiration, the fluid made its way through the muscle layer in the suture line and burst through the scar, resulting in infection of the pack space. All five had their packs removed. Three were treated by the simultaneous removal of the pack and performance of thoracoplasty in one stage. There was primary healing of the wound in two, and in both of these the sputum, which had been converted by the pack operation, remained negative and the result was highly satisfactory. The third is too recent to decide the outcome. In the other two the pack was removed and the space drained, but in both there were fatal complications. In one of them a secondary, fatal haemorrhage occurred. In the second the infection was cleared up and a seven-rib thoracoplasty carried out six weeks after removal of the pack. His lung condition was satisfactory and his sputum was negative. He had long-standing tuberculosis of the ankle joint, and, while under treatment by the orthopaedic surgeons, developed tuberculous meningitis from which he died. Postmortem examination showed widespread tuberculous deposits in the meninges, prostate, bones, and lungs. These are the only two operative deaths in the series.

In 12 patients late removal of the pack was undertaken. Six of these had developed excessive fluid formation and possibly infection in the early post-operative period, and in five of the six there was sinus formation. Repeated aspiration and injections of penicillin locally and systemically resulted in healing of the sinus and the subsidence of the infection in all. At intervals varying from five to 13 months the sinus reformed and the packs were removed. In two, after pack removal, the lung remained held down by new-formed rib under which, however, the lung cavity persisted. Thoracoplasty was carried out in three cases, with complete success in one ; with failure to achieve a negative sputum in another due to persistent cavity in the operated side, and in the third due to contralateral disease. The sixth was a patient who had had a thoracoplasty performed for a large apical cavity and subsequently a revision thoracoplasty performed which still failed to obliterate the cavity. A pack was inserted between the re-formed rib and the periosteum, but this failed to reach the cavity area. The pack was then removed and a second pack inserted directly against the lung. This resulted in cavity closure, and sputum has remained persistently negative on culture ever since. Shortly after the operation a sinus developed from which tubercle bacilli were isolated. Streptomycin injections along the track produced healing and the patient returned home. One year later 


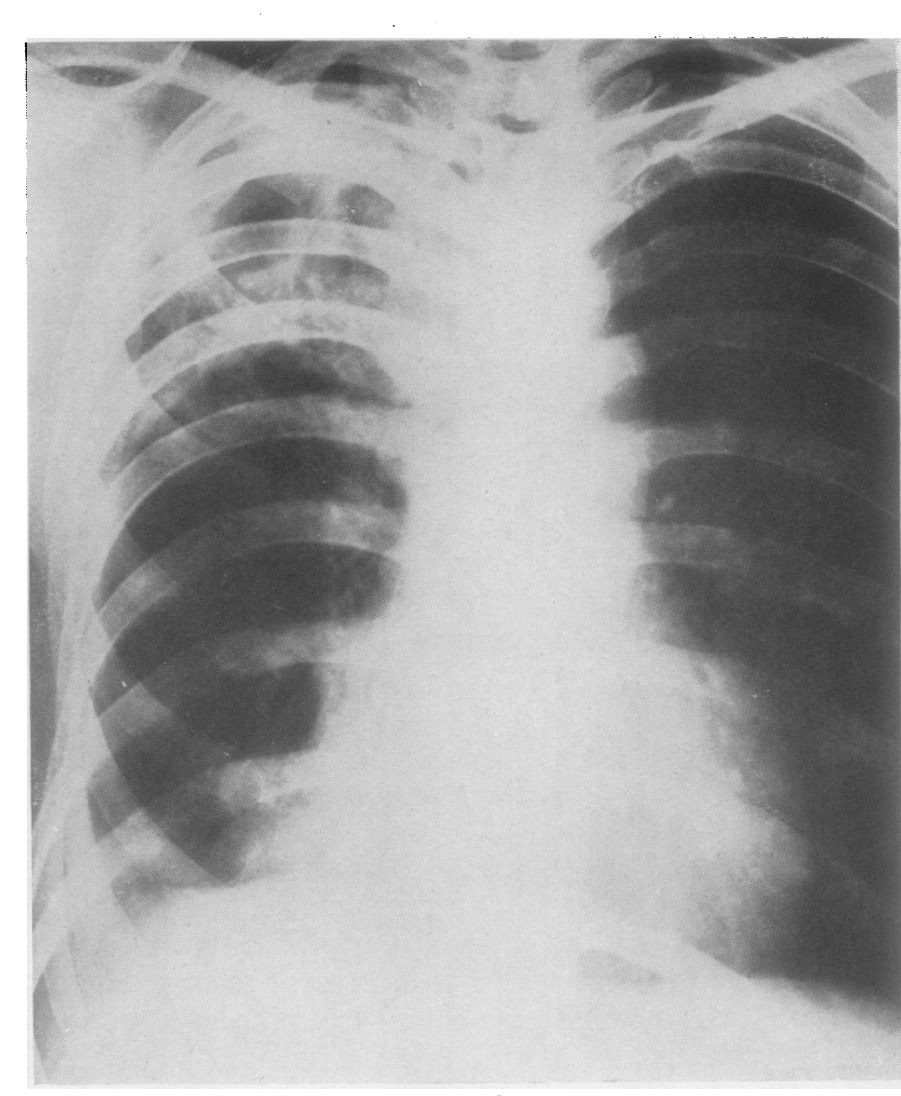

FIG. 2.

Fig. 2.-Mr. T. C., aged 34. Radiograph of March 5, 1949, showing extensive disease right apex. Sevenrib operation carried out on March 14, 1949.

FIG. 3.-The same patient. Radiograph April 6, 1949. Sputum negative and subsequently negative on culture.

FIG. 3.

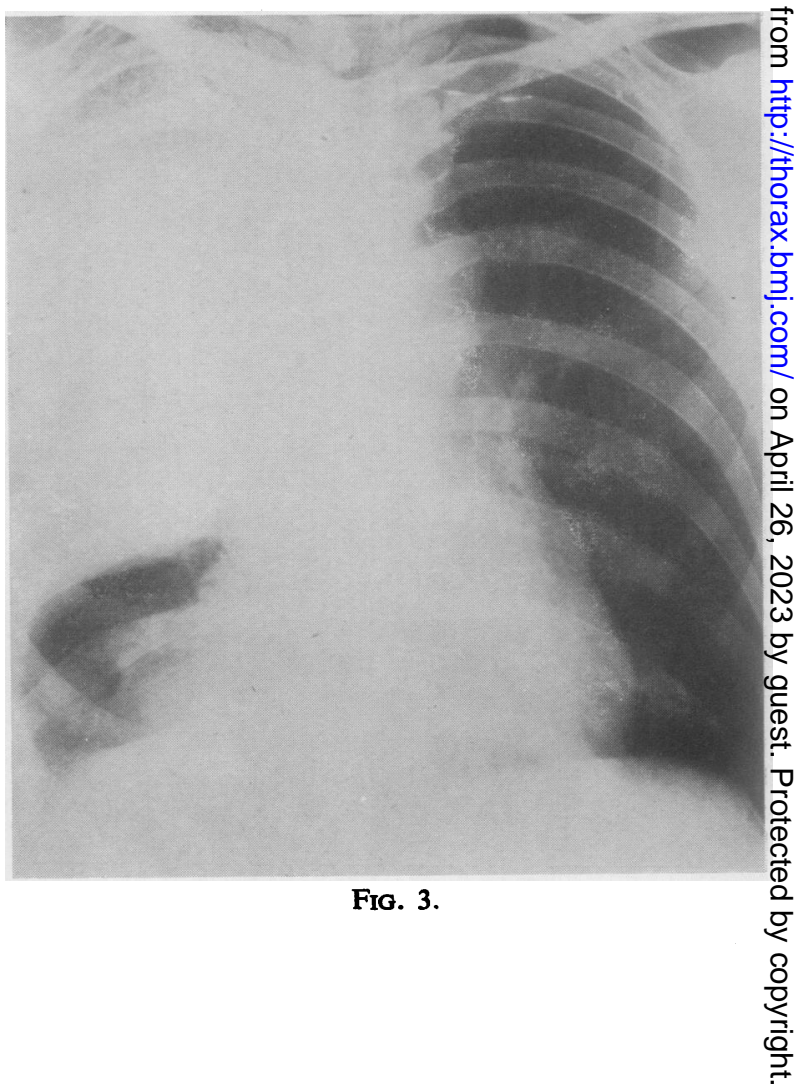



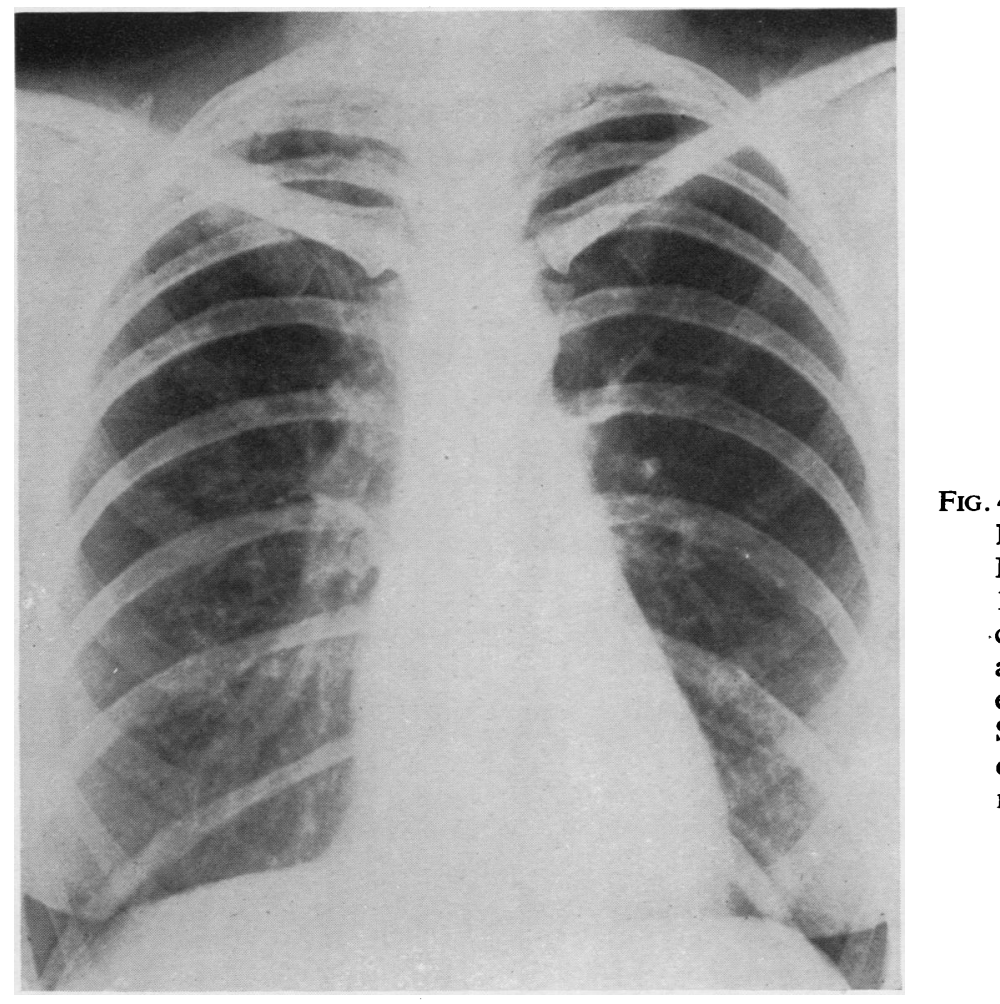

FIG. 4.

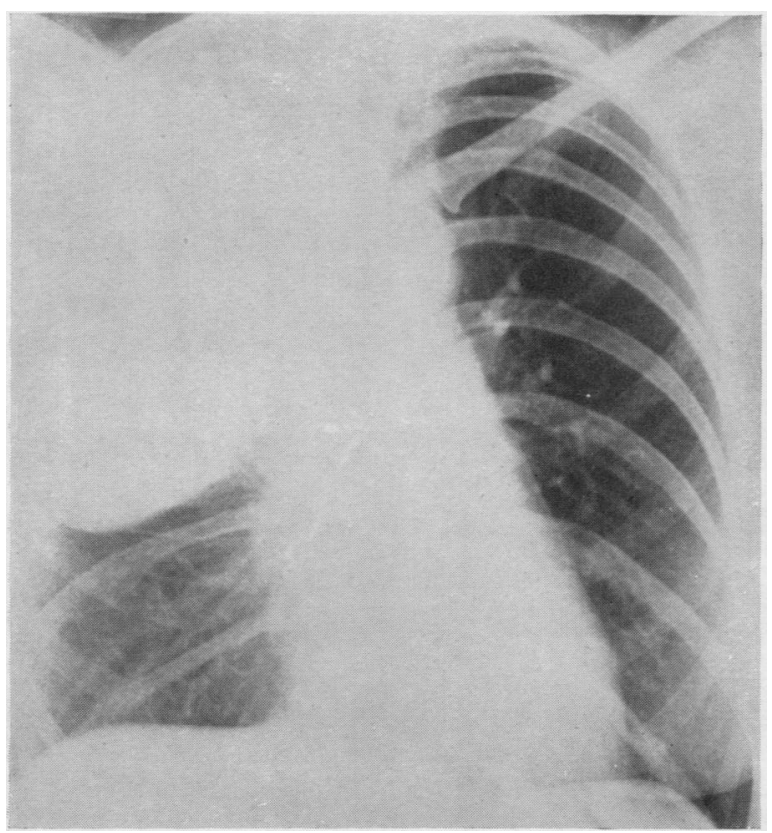

Fig. 5.

FIG. 5.-The same patient. Post-operative film, March 9, 1949.

FIG. 6.-The same patient. Lateral film March 9, 1949.

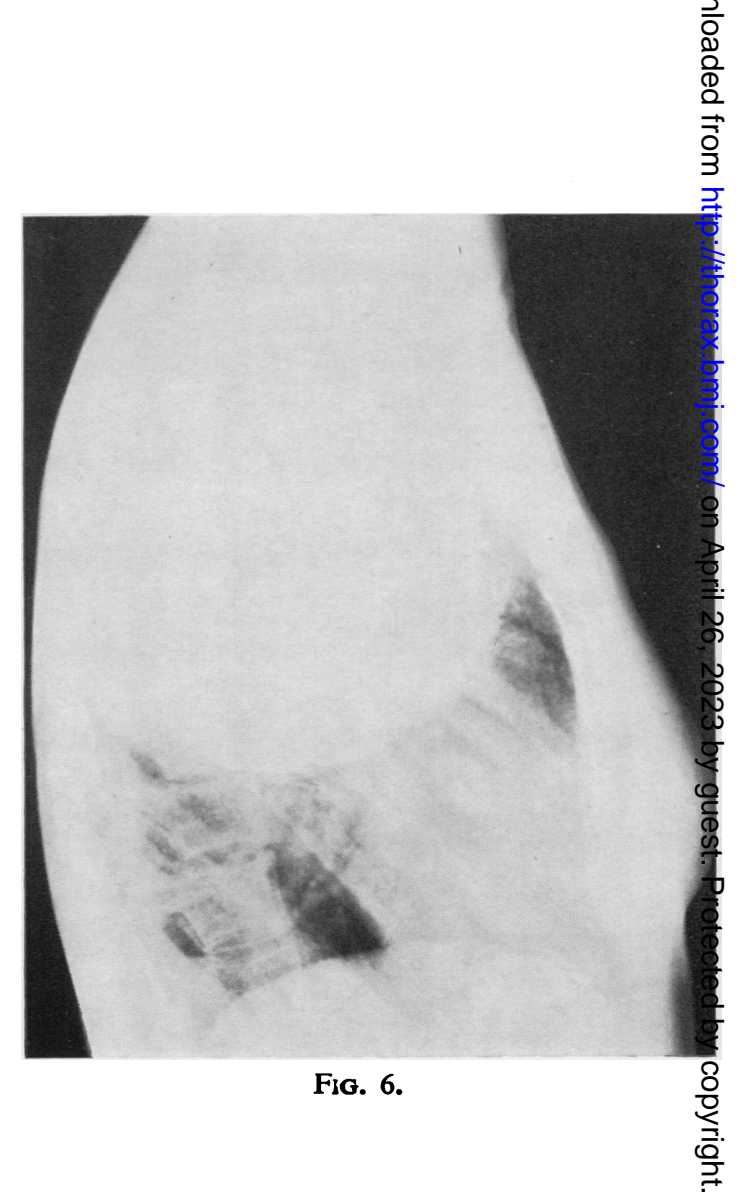


FIG. 7.

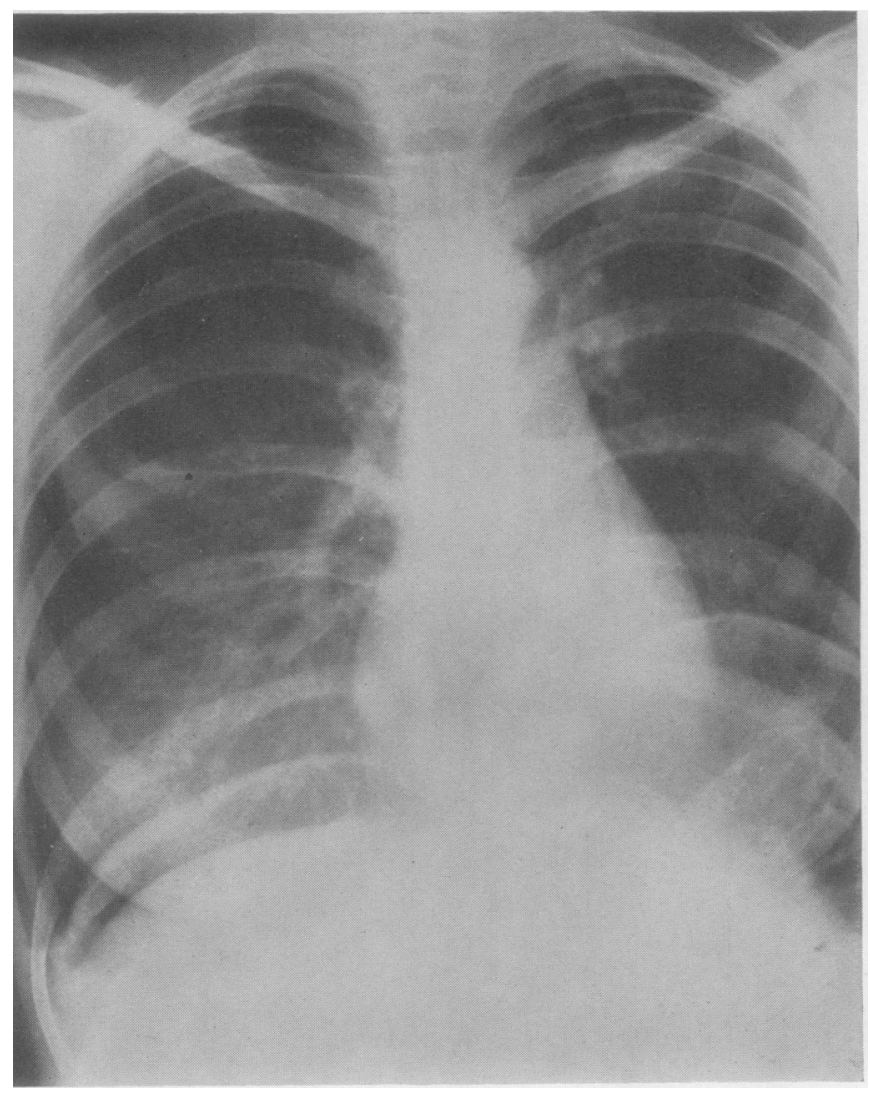

FIG. 9.

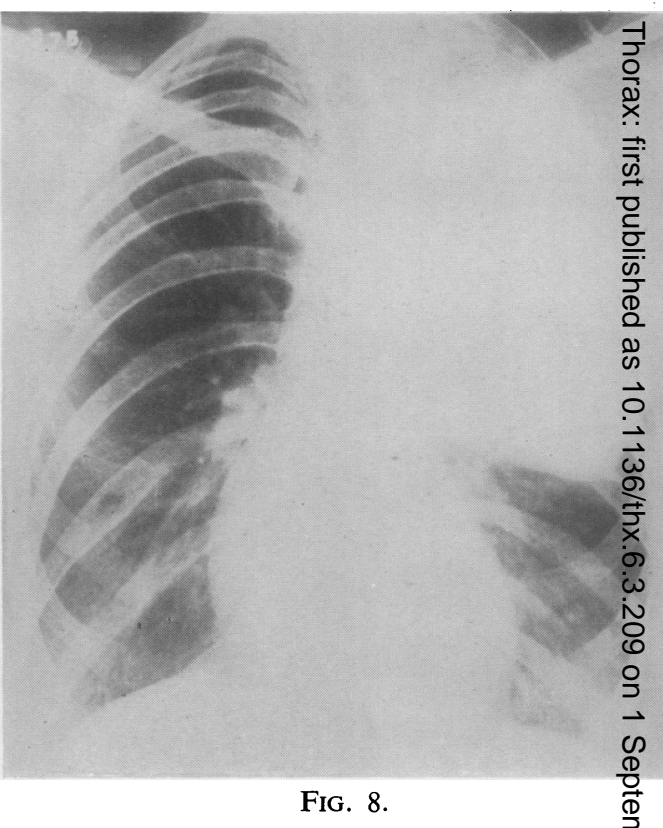

Fig. 7.-Mrs. B., aged 25. Radiograph $\frac{3}{0}$ February 8, 1949, showing extensive disease left apex. Eight-rib operation February 11, 1949.

FIG. 8.-The same patient. Radiographo April 8, 1949. Sputum culture negative

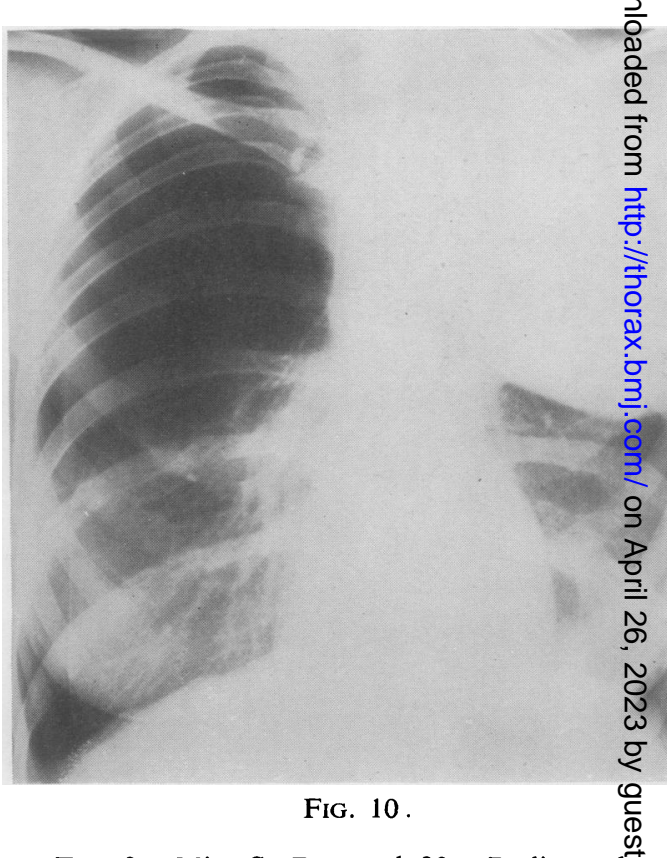

FIG. 9.-Miss S. D., aged 20. Radiograph December 13, 1948, showing right A.P. large cavity left apex, permanent lefe phrenic paralysis, and pneumoperitoneum Six-rib operation December 14, 1948.

FIG. 10.-The same patient. Radiograp April 5, 1949. P.P. abandoned, sputum negative on culture. 


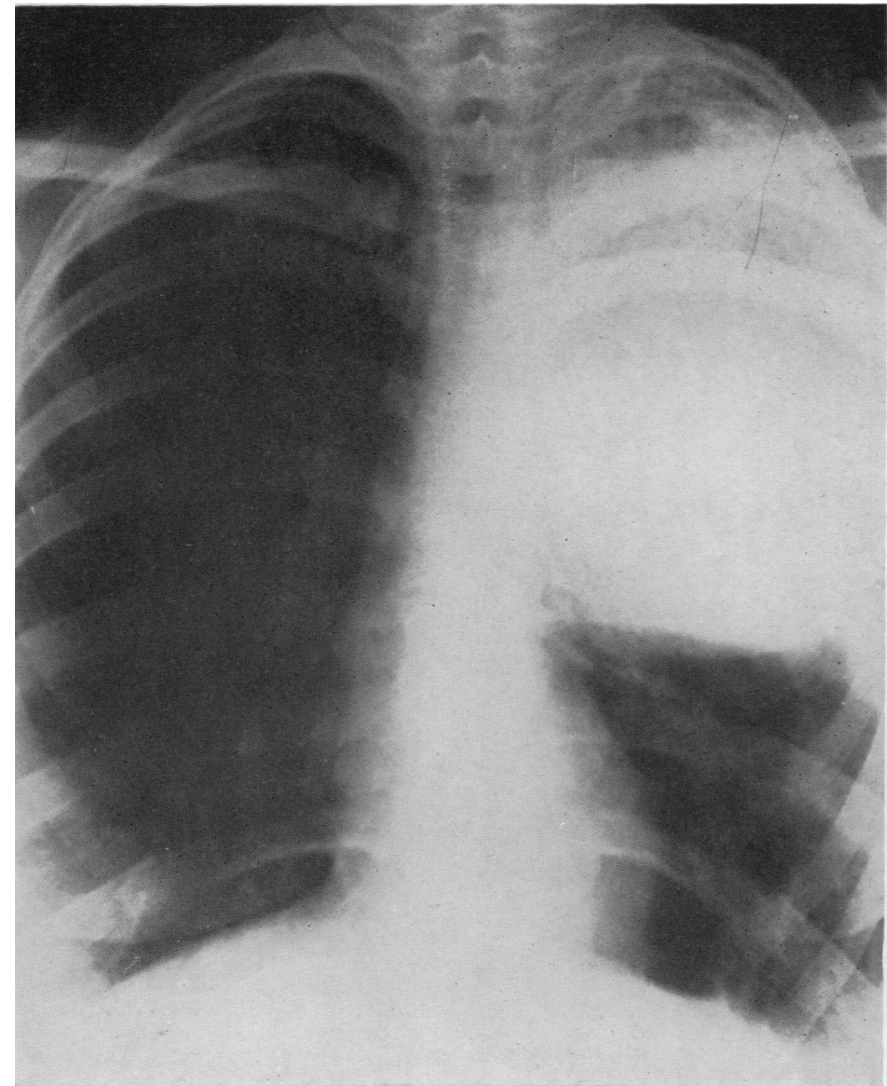

FIG. 11.

FIG. 11.-Miss M. G., aged 21. Six-rib operation April 10, 1950 . A.P. film on December 12, 1950. Sputum negative on culture.

FIG. 12.

FIG. 13.-Mr. J. C., aged 24. Six-rib operation on September 6, 1948. Bronchogram September 29, 1948, demonstrating the extent to which the upper lobe is displaced down and collapsed. This was one of the earlier cases with removal of the third rib and with lipiodol in the pack.

Fig. 12.-The same patient. Lateral film December 12, 1950.
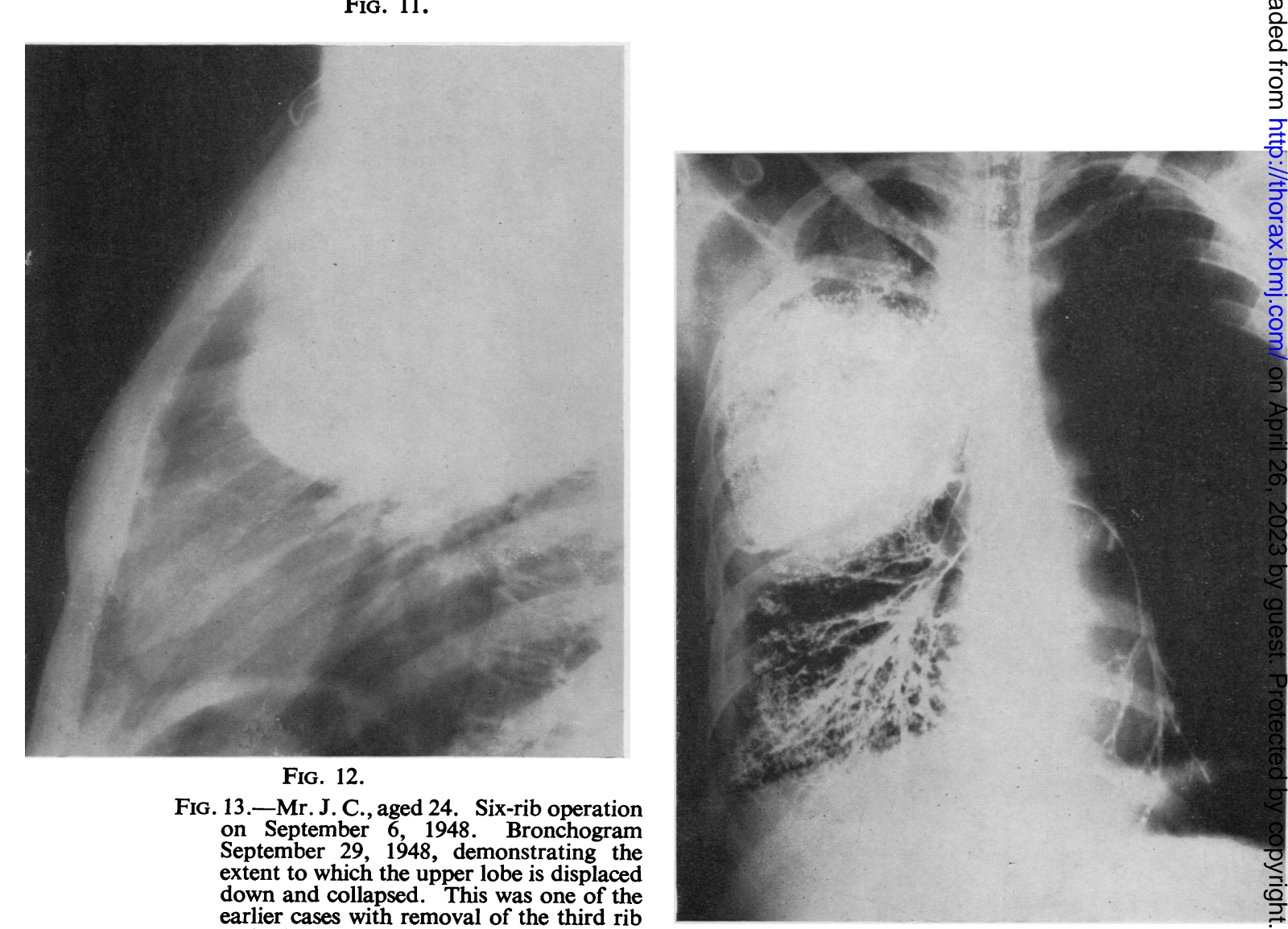

Fig. 13. 
FIG. 14.--Mr. G. W., aged 35. Film July, 1948, showing large cavity at left apex, honeycomb cavitation right apex. Eightrib left operation August 23, 1949. Fiverib right operation September 18, 1950.

Fig. 15.-The same patient. Radiograph on October 25, 1950, showing both packs in place. Sputum conversion after the second operation.

Figs. 16 and 17.-The same patient. Postoperative photographs showing free arm movements and lack of deformity.

FIG. 14.
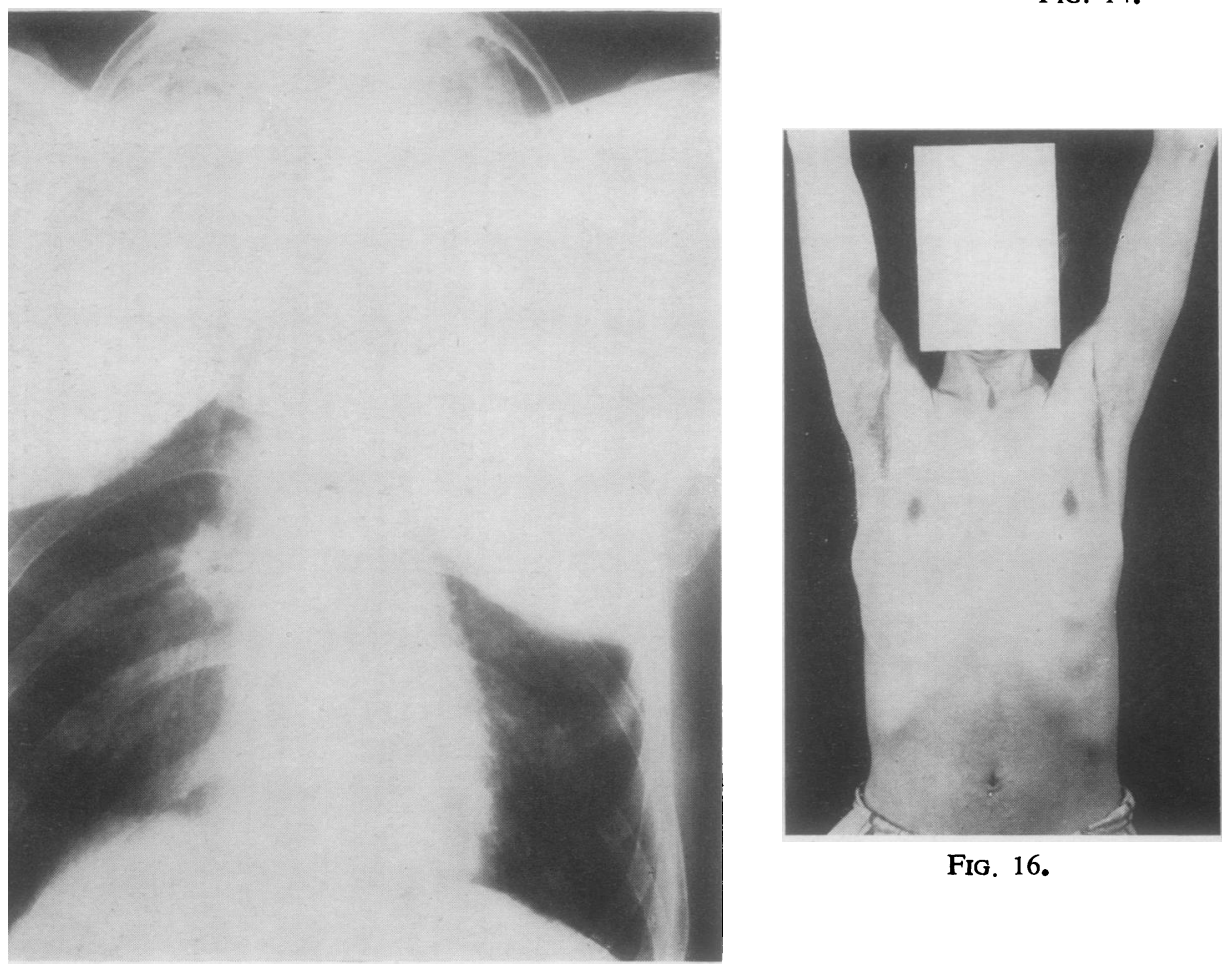

FIG. 16.

Fis. 15.

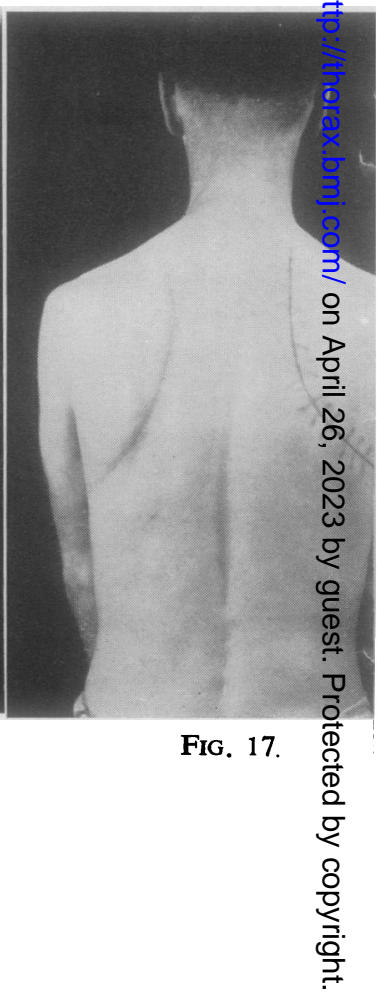


FIG. 19.

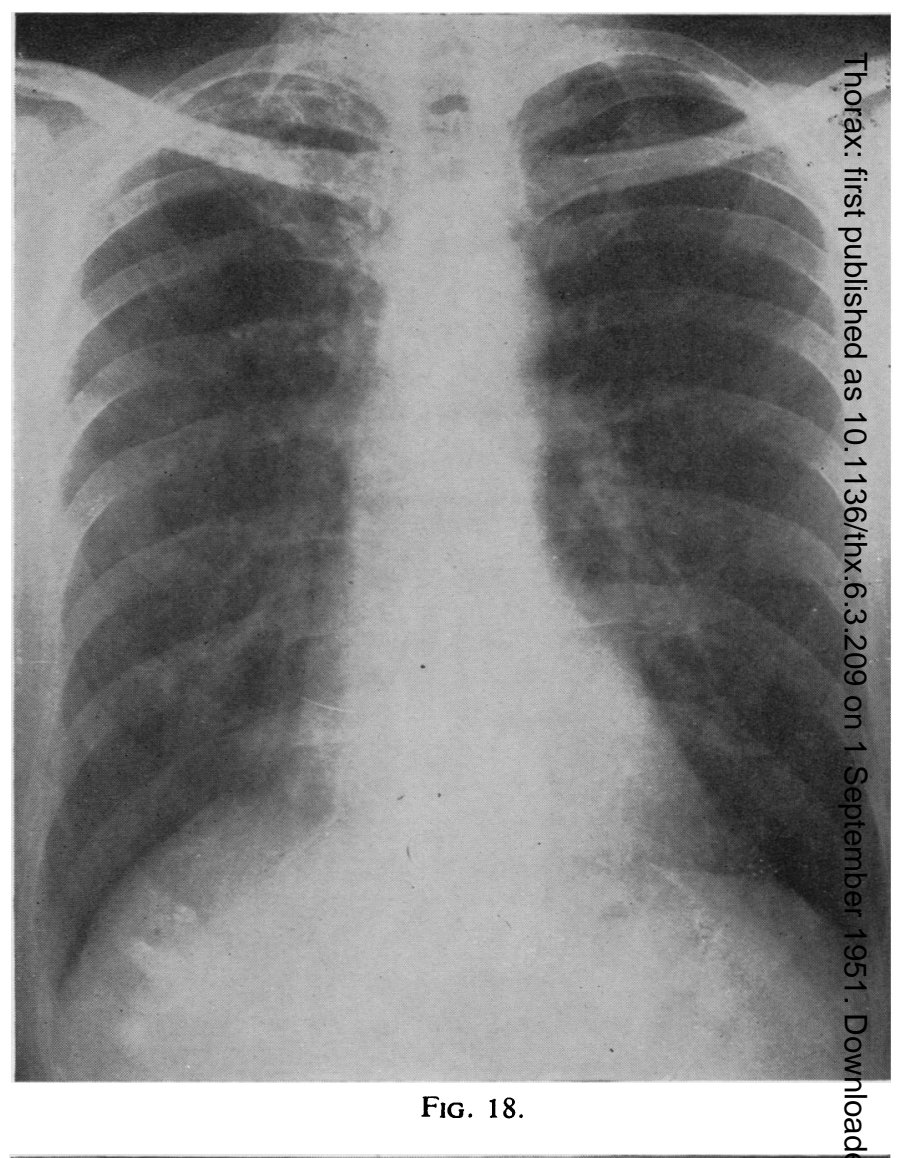

Fig. 18.-Mr. F. G. Radiograph February 17, 1950. Persistent active disease right upper lobe.

FIG. 19.-The same patient. Pack inserted February 20, 1950. Radiograph January 10, 1951. There is an infected sinus leading to pack space.

FIG. 20.-The same patient after removal of packs. There has been primary healing and the space is gradually filling with a sterile effusion. Lung remains well collapsed and sputum negative on culture. Fit for work.

Fig. 20. 
the sinus re-formed. The pack was removed and the wound healed by first intention. The lung remained adequately collapsed.

In the remaining six cases of late pack removal the immediate post-operative period following the polythene pack operation was quite straightforward and the patients were discharged. Sinuses, however, appeared at periods varying from seven to 18 months. Thoracoplasty was carried out in two cases, one with success. In the other, the pack space was found to contain tubercle bacilli, and although the thoracoplasty effectively obliterated the space there was a reactivation of the contralateral disease. The packs were removed and the space washed out and completely sutured in four. The lung was well held down with new-formed ribs and no further measures were necessary (Figs. 18-20). In three of these the sputum remained negative, and the result was satisfactory, including one case in which tubercle bacilli were isolated in the pack space at the time of removal. In the last one the contralateral disease has reactivated. Tubercle bacilli have been found in the pack space in four cases. In the three already mentioned the infection appeared to be benign and responded promptly to treatment. In the fourth it occurred on a single occasion. After local streptomycin injection the lesion has remained healed and the sputum negative for 13 months. The pack was not removed.

There were no other complications, and there was no evidence of pressure on the mediastinal veins and no suggestion of ulceration of the pack into the lung or from the lung into the pack space. Further, there was no evidence of necrosis of the ribs in the absence of infection. Since resection of ribs was abandoned there has been no instance of displacement of the pack post-operatively.

\section{CONCLUSIONS}

A review of these complications shows that the outstanding one which detracts from the otherwise somewhat impressive merits of the operation is that of sepsis. Subtracting those cases of the earlier group in which ribs were resected, which later allowed the partial herniation of the pack, the irritation of the scar, and a secondary infection from outside, most of the remaining cases of infection are associated with excessive fluid formation. There is the possibility that in some cases the excessive fluid formation was stimulated by infection. Although in a few cases this may have been so and cannot be disproved, in the majority the fluid was at first sterile by all ordinary tests and infection occurred secondarily to it, being a retrograde contamination from the skin. We are unable to find, as yet, an adequate explanation for excessive fluid formation. It has occurred in cases operated on by all of us ; in cases that have bled freely or been comparatively dry; in cases that have been difficult or easy ; when the extra-fascial strip has been $1 \mathrm{~cm}$. only in extent or much more extensive; when the packs have been sterilized by formalin or boiled; immaterially whether open or closed packs have been used; whether penicillin tablets have been left in the wound or procaine penicillin; whether the space has been packed tightly or without undue pressure. Moreover, there has been nothing in the nature of the disease in any particular case, in the site of the cavities, or in previous pleural incidents, to give us a clue. We feel, none the less, that the more the pliability of the polythene is maintained the less tendency there is for irritation or trauma of the tissues. It is for this reason that lipiodol is included in the packs. 
Approximately one-sixth of the patients were women. It may be of no significance, yet it is interesting to note that, apart from one case with a cavity in the apicolower segment which became septic, there was no other incidence of sepsis or of excessive fluid formation.

In May, 1948, a preliminary report on the operation was made in the course of a paper read to the Thoracic Society (Morriston Davies, 1948). The operation has been taken up by a number of surgeons in this country and abroad. Since then, technique has been altered and certain mistakes eliminated, and it is for this reason that we feel we should set forth the latest details of the preparation of the packs and of the operation and of our mistakes, our successes and our failures, as they may be of value to those interested. Only the passage of time can test the value or otherwise of a new type of operation such as this, particularly when it combines the introduction among the body tissues of a foreign body - that anathema of all surgeons. Two and a half years is, in our opinion, too short a time, especially as it is less than six months since we made our latest major alterations in the technique.

One big mistake made at the beginning, and for some months afterwards, was the removal of a certain number of ribs to facilitate access to the first and second ribs and for the insertion of the packs. It allowed of a partial herniation of the packs through the spaces thus left between the ribs. This, combined with the fact that we used deliberately to leave a considerable amount of air with the polythene strips in the unsealed packs, resulted in some diminution of the intrathoracic space occupied by the packs and allowed a slow, partial re-expansion of the collapsed lung. The process was so gradual that in most cases it did not matter, as the lung lesions had healed, but not always. Another and more serious result, however, of the herniation of the packs was that they came into contact with the soft tissues and caused bulging and ulceration of the scar and infection of the pack and pack space as has already been noted.

Another mistake, which should also have been foreseen, was that the first two cartilages only were cleared, whereas it is now evident that the cartilages of the first three ribs must be exposed as far forwards as possible. Equally so is the importance of dividing the intercostal muscles and neurovascular bundles as far back as possible so as to ensure that the packs can be bedded down into the costovertebral gutter.

Our experience has shown that certain types of disease are not suitable for treatment with a pack: pre-eminently among these are cavities in the lower lobe, whether in the apico-lower or in the basal segments. Lungs in which the apex is shelled out and there is a large tension cavity should obviously be treated by resection. If for various reasons this is contraindicated and, despite contralateral disease, a collapse operation is feasible a thoracoplasty is preferable to a pack. A pack operation cannot be done following the insertion of a Monaldi catheter, but it is possible after cavity reduction by posture. Cavities with very thick walls are unsuitable. No type of tuberculoma is suitable, in our opinion, for any collapse treatment.

The modern trend of surgery in the treatment of pulmonary tuberculosis is such that what used to be regarded as the ideal case for a thoracoplasty and would be regarded still more so for a polythene pack, since there is no associated deformity, is now, par excellence, considered the ideal for a lobectomy. The polythene pack operation has to be reserved for such cases in which the resection is considered 
unsuitable ; cases, that is, in which the existence of disease, past or present, in the opposite lung makes the operation of lobectomy or of pneumonectomy less advisable or not to be considered. This selection and reservation for resection of the more ideal cases of tuberculosis - those with minimal or localized disease and definitely not bilateral active lesions-is obviously an important consideration to be taken into account when estimating and comparing the results of the various operations. Another difficulty in assessing the results of resection and of the pack operation is that, while the long-term results of resection are comparatively few, those of the pack are non-existent.

We are in a position to make certain claims for the advantages of the pack operation over the thoracoplasty operation. The polythene pack operation, since it removes no ribs, produces no deformity. The third rib which has been divided at the beginning of the operation is, if correctly sutured, entirely reconstituted. Pain is almost absent, and the physiotherapy necessary to free the scapula from the restraint of sutured muscles is minimal. The necessary aftercare by the physiotherapists and nursing staff is even less than after a resection. When compared with a thoracoplasty of considerably less degree the difference is the more striking. Not only is there comparative absence of pain, but still more noticeable is the minimal shock even if a one-stage eight-rib pack is compared with a first-stage three-rib thoracoplasty. This is due to the absence of any "mediastinal flutter" during and after the insertion of the pack and, also, the great difference in pain. The anaesthetists have repeatedly commented on the ease with which a patient tolerates a six-, seven-, or eight-rib pack operation in one stage. It is hardly necessary to menticn the mental gain to the patient who, needing the collapse of the greater part of his lung, is submitted to a single operation instead of the three stages entailed by a thoracoplasty. Moreover, in a multiple-stage thoracoplasty the associated risks of a spill-over of secretion into the contralateral lung and of post-operative atelectasis are necessarily greater, and with these are the anxieties and hazards due to the extended delay between the stages.

\section{Other Uses of the Polythene Pack}

Once the value of the polythene pack in effecting a permanent collapse of a diseased lung had been established, the possibility that this principle could be made use of in other conditions was considered. The first attempts were made in two cases of empyema, but both failed. The disease had been of long standing and the pleura was so thickened that it was unyielding.

The next endeavour was to prevent hyperexpansion of the contralateral lung after a pneumonectomy. The operation and immediate result were satisfactory, but the patient died one year later from Hodgkin's disease. The findings at necropsy as regards the pack space were most encouraging (Fig. 1).

A pack was used with a similar object in a patient with bronchiectasis, in whom the maximal changes were in the left lower lobe but minor bronchiectatic changes were also present elsewhere. The lower lobe was removed, and two weeks later, in order to prevent hyperexpansion of the remaining lung, an extra-pleural-periosteal pack was inserted in the lower part of the chest. The result was entirely satisfactory.

The next problem tackled was that of empyema and bronchopleural fistula following pneumonectomy: three such cases were operated on. In every case the 
empyema was drained anteriorly and the packs put in posteriorly. The bronchopleural fistula was immediately and permanently closed in all three and the empyema space obliterated. One patient who was operated on in September, 1948, has remained quite well and is doing full work. In the other two, at varying periods after the operation, the pack space became infected with the same organisms that had been present in the empyema. The pack was removed and a thoracoplasty done.

Recently, with the greater use of pulmonary resection in tuberculosis, the problem of diminishing the empty hemithorax and preventing hyperexpansion of the opposite lung has become increasingly important. The rise of the hemidiaphragm as a result of a phrenic crush and the temporary pneumoperitoneum diminishes the hemithorax to a considerable extent, but there is still a large space into which the opposite lung will herniate. In four cases, following a pneumonectomy, a polythene pack operation was done on the upper thorax. The operation was quickly and easily carried out and had little disturbing effect on the patient. There have been no post-operative complications: the mediastinum has remained central, and there is no resulting deformity. It is worth considering whether this should not be a routine procedure two to three weeks after a pneumonectomy for pulmonary tuberculosis.

Even when the-lung had been removed with the adherent parietal pleura, the subsequent operation for the insertion of the pack presented no difficulties as, in the intervening weeks, the periosteum had become reinforced by a layer of fibrin. In one case only was the pleural space opened, and as there was no infection of the space no ill results followed.

We gratefully acknowledge our indebtedness to Dr. L. B. Wevill, Dr. A. S. R. Stewart, and to Mr. S. Holt, of Imperial Chemical Industries, Ltd., for their constant advice, interest, and generous help, including the supply of polythene. The experimental work was made possible only by the kindness and help of Professor C. A. Wells and Mr. F. Ronald Edwards, of the Department of Surgery, University of Liverpool. To the medical and nursing staffs of the Liverpool Thoracic Surgical Centre, Broadgreen Hospital, and the Cheshire Joint Sanatorium, we are greatly indebted for their interest and assistance. We offer our thanks to Dr. Robert Coope for his criticism and advice in preparing this paper.

\section{BIBLIOGRAPHY}

Bailey, C. P. (1942). J. thorac. Surg., 11, 326.

Brown, M. H., Grindlay, J. H., and Craig, W. McK. (1948). Surg. Gynec. Obstet., 86, 663.

Davies, H. Morriston (1933). Pulmonary Tuberculosis: Medical and Surgical Treatment, pp. 411420. London. (1944). Brit. med. J., 2, 1145.

(1948). Thorax, 3, 189 .

Edwards, F. R. (1949). Ibid., 4, 224.

Farquer, J. W., and Lewis, I. C. (1948). Lancet, 2, 244.

Grindlay, J. H. (1948). Surgery, 24, 22.

Ingraham, F. D., Alexander, E., and Matson, D. D. (1947). J. Amer. med. Ass., 135, 82.

Lucas, B. G. B., and Cleland, W. P. (1950). Thorax, 5, 248.

Paneth, O. (1946). Brit. J. Tuberc., 40, 60.

Semb, C. (1937). Brit. med. J., 2, 650. (1950). Personal communication.

Trent, J. C., Moody, J. D., and Hiatt, J. S. (1949). J. thorac. Surg., 18, 173.

Wilson, D. A. (1946). Surg. Clin. N. Amer., 26, 1060.

Yeager, G. H., and Cowley, R. A. (1948). Ann. Surg., 128, 509. 